\title{
A Comparison between Piezoelectric Sensors Applied to Multiple Partial Discharge Detection by Advanced Signal Processing Analysis ${ }^{+}$
}

\author{
Amanda Binotto ${ }^{1,2, *} \mathbb{D}^{D}$, Bruno Albuquerque de Castro ${ }^{1}\left(\mathbb{D}\right.$, Vitor Vecina dos Santos ${ }^{1}$, \\ Jorge Alfredo Ardila Rey ${ }^{3}$ (D) and André Luiz Andreoli ${ }^{1}$ (D) \\ 1 Department of Electrical Engineering, School of Engineering, São Paulo State University (UNESP), \\ 17033-360 Bauru-SP, Brazil; bruno.castro@unesp.br (B.A.d.C.); vitor.vecina@unesp.br (V.V.d.S.); \\ andre.andreoli@unesp.br (A.L.A.) \\ 2 IEEE Women in Engineering, São Paulo State University (UNESP), 17033-360 Bauru-SP, Brazil \\ 3 Department of Electrical Engineering, Universidad Técnica Federico Santa María, \\ Av. Vicuña Mackenna 3939, Santiago de Chile 8940000, Chile; jorge.ardila@usm.cl \\ * Correspondence: amanda.binotto@unesp.br \\ + Presented at the 7th Electronic Conference on Sensors and Applications, 15-30 November 2020; \\ Available online: https://ecsa-7.sciforum.net/.
}

Published: 15 November 2020

\begin{abstract}
The development of sensors applied to failure detection systems for power transformers is a critical concern since this device stands out as a strategic component of the electric power system. Amongst the most issues is the presence of partial discharges (PD) in the insulation system of the transformer which can lead the device to total failure. Aiming to prevent unexpected damages, several PD monitoring approaches were developed. One of the most promising is the Acoustic Emission (AE) technique which captures the acoustic signals generated by PDs using piezoelectric sensors. Although many studies have proved the effectiveness of AE, most signal processing approaches are strictly related to the frequency analysis of PD signals, which can hide important information such as the repetition rate of the failure. This article presents a comparison between two types of piezoelectric transducers: the micro fiber composite (MFC) and the lead zirconate titanate (PZT). To ensure the detection of multiple PDs the time-frequency analysis was carried out by Short-time Fourier transform (STFT). Intending to compare the sensibility of the transducers, the AE signals were windowed, and the root mean square (RMS) value was extracted for each part of the signal. Results indicated that spectrogram and RMS analysis have great potential to detect multiple PD activity. Although MFC was 2 times more sensitive to PD detection compared with the PZT sensor, PZT presents a higher frequency response band $(0-100 \mathrm{kHz})$ concerning MFC $(80 \mathrm{kHz})$.
\end{abstract}

Keywords: piezoelectric sensors; partial discharges; transformers diagnosis; time-frequency analysis; acoutic emission

\section{Introduction}

Power Transformers are essential electrical distribution equipment since they are responsible for the adequacy of voltage levels and energy transference from generating units to consumption points [1]. Some concerned reasons in the operation of transformers, for instance, overheating, overload operation, harmonics produced by switching, and nonlinear loads can damage the insulation system of transformers, culminating with the appearance of partial discharges $[2,3]$.

Partial discharges are an aleatory phenomenon that emits UHV, heat, electromagnetic and acoustic waves. The more it has its occurrence, the more impaired is the insulation system $[4,5]$. Therefore, PD is both evidence and cause of the insulation system degradation, and this phenomenon can lead 
the transformer to a complete loss. Consequently, power transforms supervision, and monitoring is convenient to avoid and prevent failures. There are many methods to locate and identify PD, such as chemical, electrical, electromagnetic, and acoustic [3,6-9].

However, many methods are invasive, i.e., they depend on actions, such as shutdowns, disassembly, removal from site. On the other hand, some methods, like the electric, may expose people to high voltage levels since the PD detection relies on the input and output voltage analysis of the transformer $[6,10]$.

In this sense, the Acoustic emission (AE) method is characterized as a non-invasive methodology that captures ultrasound waves emitted by PDs $[9,11]$. The preventive diagnosis can be made with the device in operation by attaching AE sensors in the transformer's external wall, aiming to detect acoustic waves produced by PD activity $[2,8]$.

Although many studies have proved the effectiveness of AE, most signal processing approaches are strictly related to the frequency analysis of PD signals, hiding important information such as the failure's repetition rate [12]. In addition, there is a need for the development and validation of other types of transducers to expand the applicability of this type of non-destructive testing (NDT). Experiments show that low-cost sensors, as piezoelectric diaphragms, commonly known as buzzers, have feasibility for detecting partial discharges under the experimental conditions [2]. However, one disadvantage to overcome is the minor development of low-cost commercial AE sensors to PD detection.

In this context, this paper presents a comparative study between two low-cost piezoelectric transducers: the microfiber composite (MFC) P1 type [2], and the lead zirconate titanate (PZT), either analyzed by two signal processing metrics, the root mean square (RMS) value and Short-time Fourier transform (STFT) [12]. These metrics were extracted intending to compare the transducers' sensibility and ensure the detection of reoccurring PDs. Results indicated that spectrogram and RMS analysis have great potential to detect multiple PD activity, though MFC was more susceptible to PD detection than the PZT sensor.

This paper is organized as follows: Section 2 presents the AE method for PD detection. The signal analysis applied in this article is discussed in Section 3. The experimental setup is described in Section 4, and then, in Section 5, the results are presented and discussed. Section 6 reports the conclusions of this article.

\section{AE Method for PD Detection}

Previously to the total failure of transformers, it is common to detect low energy ionization processes in the insulation system of the device. This phenomenon, known as partial discharge (DP), is defined by the IEC 60270 (2000) as an electrical discharge that short-circuits only a part of the insulating material [13]. PDs produce pulses of current, heat, electromagnetic waves, ultraviolet radiation, and acoustic waves, increasing the material's degradation [10,14-17].

Among several approaches, PD acoustic emission-based detection is a promising technique that applies piezoelectric transducers to the transformer wall to capture acoustic waves emitted by the failure. However, current challenges are related to the development and validation of other types of piezoelectric transducers aiming to expand the applicability of this type of non-destructive testing (NDT). This article carried out a comparison between two types of low-cost piezoelectric transducers: the microfiber composite (MFC) M2814-P1 type, and the lead zirconate titanate (PZT). In relation to PZTs, MFCs are flexible devices allowing its attachment to many uneven surfaces of the transformer, which would not be accessible with conventional sensors.

Besides the validation of piezoelectric transducers, it is crucial to develop signal processing analysis to perform the correct failure feature extraction. The next section discusses the signal analysis applied to compare the transducers used in this work.

\section{Digital Signal Processing Analysis}

Most signal processing approaches applied to PD detection are strictly related to the frequency analysis of PD signals, which can hide important information such as the failure's repetition rate. 
Based on this issue, to ensure the detection of multiple PDs, the time-frequency analysis was carried out by Short-time Fourier transform (STFT). The STFT is a digital signal processing technique that segments a signal into a set of short subsequences centered on uniform time intervals[12]. For each interval, the discrete Fourier transform was calculated separately, according to Equation (1) [18]:

$$
\operatorname{STFT}(t, \omega)=\int h(u) f(t+u) \cdot e^{-j \omega u} d u,
$$

where $f(t)$ is a signal in the time domain, $t$ is the time, $\omega$ the frequency, and $h(u)$ is window function set Gaussian.

Intending to compare the transducers' sensibility, the AE signals were segmented by rectangular windows with length $T$, and the root mean square (RMS) value was extracted for each part of the divided signal. RMS value is defined according to Equation (2) [19]:

$$
R M S=\sqrt{\frac{1}{T} \int f(t)^{2} d t} .
$$

\section{Experimental Setup}

The MFC-P1 (M2814-P1) and PZT (7BB-35-3) sensors were fixed to a 30 kVA transformer wall (Figure 1) using liquid paraffin to capture the AE signals emitted by partial discharges. The acquisition rate was set $10 \mathrm{MHz}$, and the signals were amplified 25 times by the INA 128P (Texas Instruments®). As the amplifier has a frequency response until $400 \mathrm{kHz}$, it was used as an anti-aliasing filter. An electrode with a $5 \mathrm{~mm}$ gap generated PD activities by an application of $3.5 \mathrm{kV}$. All cables were grounded to avoid electromagnetic interference. To mitigate vibration effects, a high pass digital filter was applied with a cutoff frequency of $20 \mathrm{kHz}$. Several routines for signal processing analysis were developed in the Matlab ${ }^{\circledR}$, such as RMS value and Short Time Fourier Transform. The window used to calculate RMS had 5000 points, and the temperature of the test remained constant.

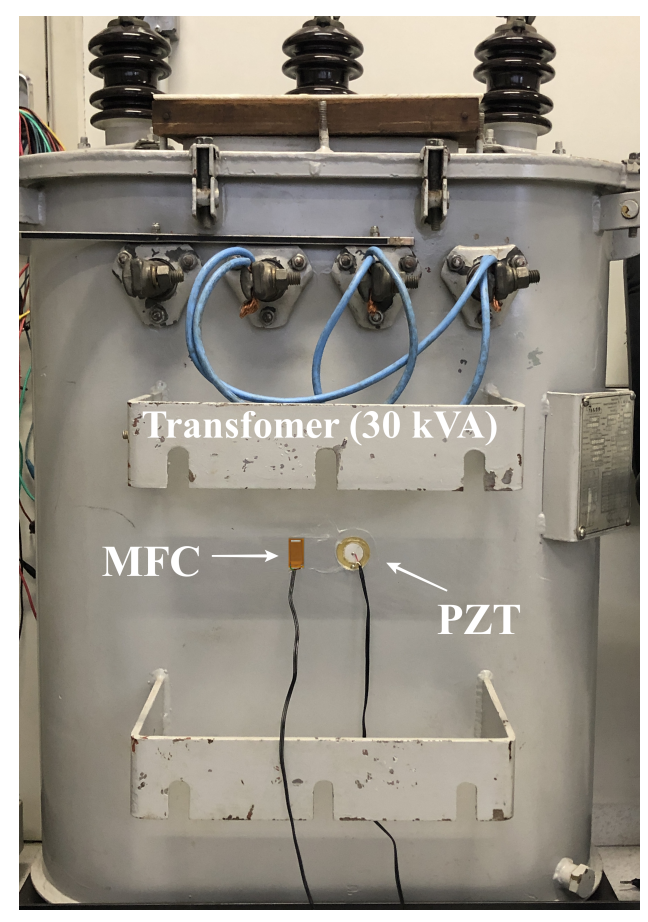

Figure 1. Transducers attached to transformer.

\section{Results and Discussion}

This section performs the comparative analysis of two low-cost sensors in time and frequency domain. 


\subsection{Time Domain Response}

A study of PD signals was achieved in the time-domain, to examine the sensibility of the transducers. Even though this simple interpretation is not suitable to estimate the sensor's usefulness, it supports the features extraction of the signals such as RMS value and power spectrum density. Figure 2a,b show the discharge signals collected by the PZT and the MFC sensors, respectively.

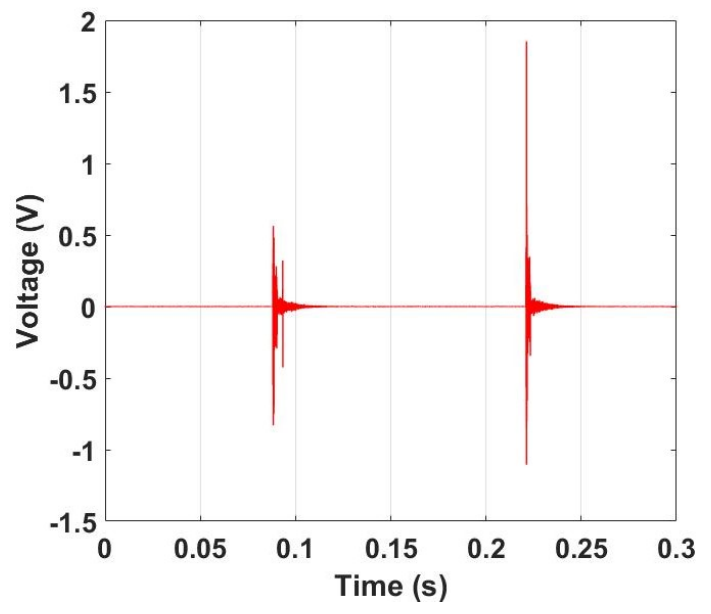

(a)

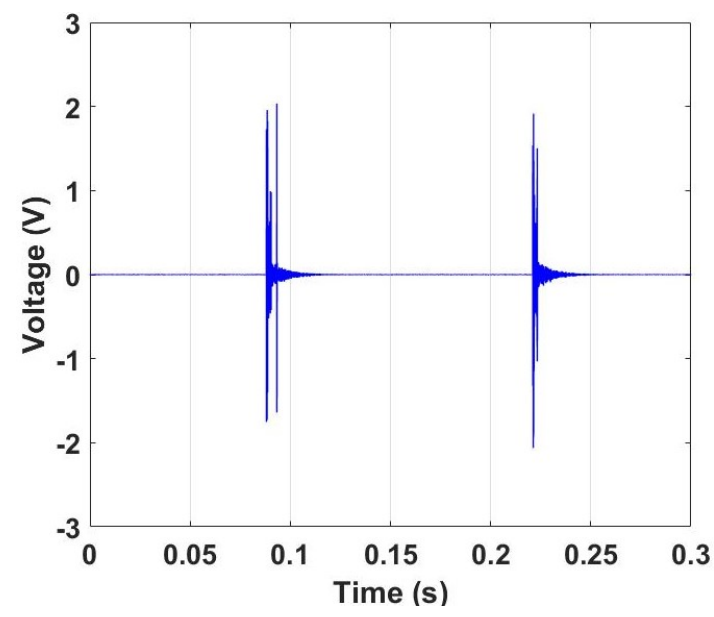

(b)

Figure 2. Raw PD acoustic emission signals for: (a) PZT and (b) MFC sensor.

It is possible to notice that both sensors detected the acoustic emission of two PD occurring. Even though there are contrasts in amplitude between the two waveforms in the time domain, mainly in detecting the first DP occurrence, both signals have correlated tendency. The two signals abruptly rise approximately in $0.086 \mathrm{~s}$ and reduce in relatively $0.11 \mathrm{~s}$ for the first PD; the second PD detected signals increase, once more, in $0.22 \mathrm{~s}$ and $0.248 \mathrm{~s}$.

Figure 3a show the RMS values collected from the PZT sensor and Figure 3b from the MFC sensor.

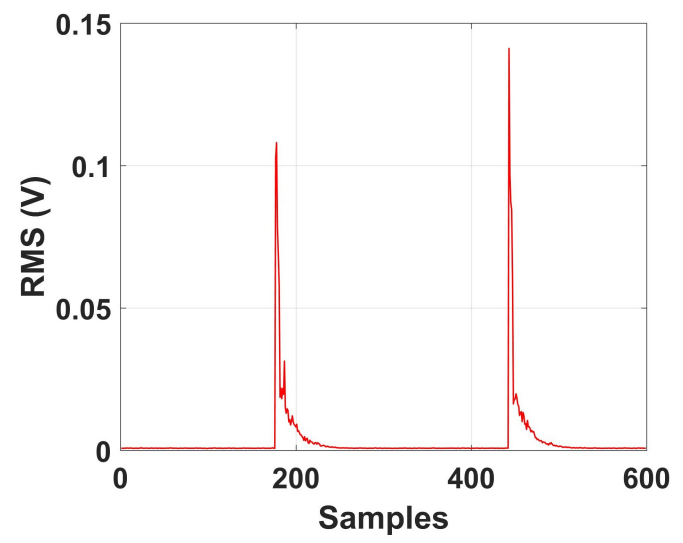

(a)

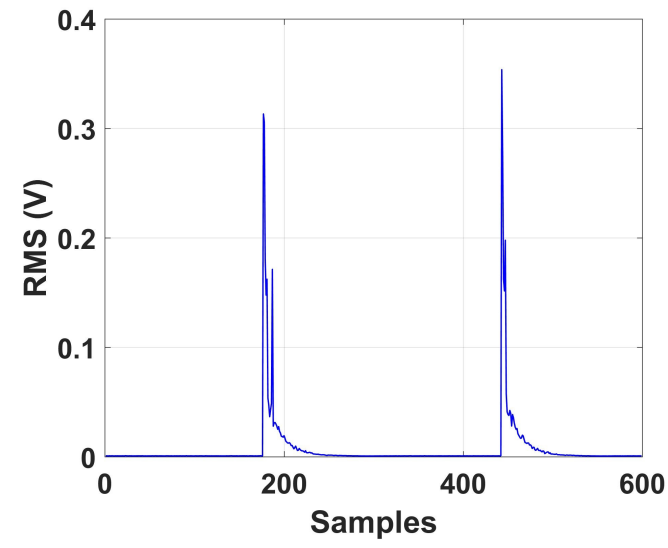

(b)

Figure 3. RMS values of the partial discharge signal for: (a) PZT sensor (b) MFC sensor.

Results show that the RMS parameter has great potential to detect multiple PD, once the graphic presents two peaks of each PD activity. Furthermore, by RMS analysis, it is possible to observe that the MFC sensor has higher sensibility than PZT. The first peak value analyzed for the MFC sensor was $0.313 \mathrm{~V}$; meanwhile, this parameter was $0.108 \mathrm{~V}$ for PZT. By analyzing the second DP, the amplitude was $0.141 \mathrm{~V}$ for the PZT and $0.354 \mathrm{~V}$ for the MFC sensor. Due to material stress caused by the first failure, the second PD presents higher values of RMS. 
By extracting the average of the RMS vector, outcomes show that for the PZT sensor, this value was $0.0033 \mathrm{~V}$, and for MFC, $0.0074 \mathrm{~V}$. Therefore, it can be concluded that MFC was 2.22 times more sensitive to PD detection than the PZT sensor.

\subsection{Frequency Domain Response}

To guarantee the observation of recurring PDs and analyze the frequency response band, STFT was extracted. Figure 4a,b present the STFT for the PZT and the MFC sensors, respectively.

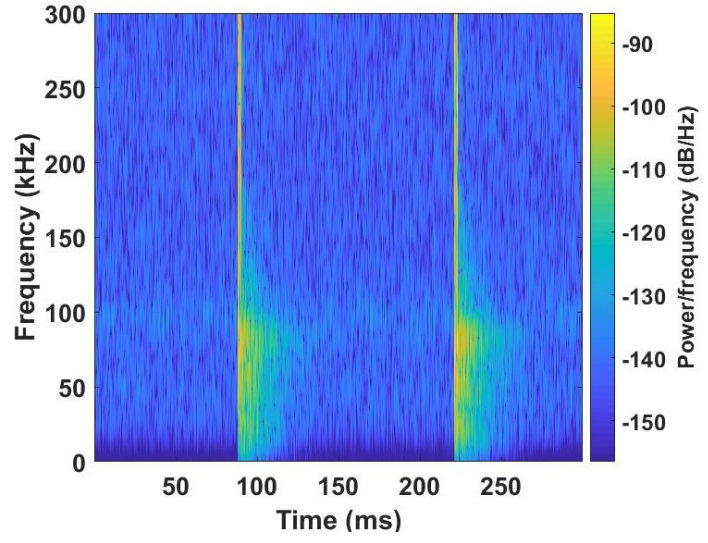

(a)

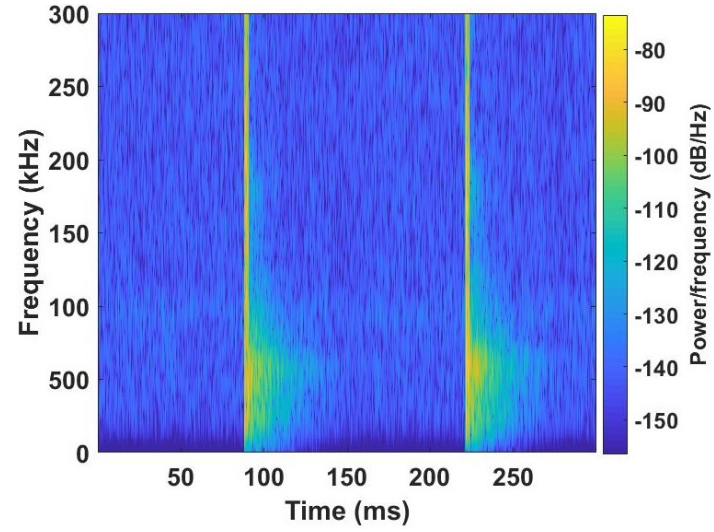

(b)

Figure 4. Spectrogram for: (a) PZT sensor and (b) MFC sensor.

By analyzing the power spectrum density values until $115 \mathrm{~dB} / \mathrm{Hz}$, it can be concluded that the PZT sensor has a higher frequency response band varying from 0 to $100 \mathrm{kHz}$, opposed to MFC, which presented a frequency band until $80 \mathrm{kHz}$.

Finally, it is important to note that the outcomes registered that spectrogram and RMS analysis have great potential to identify reoccurring PD activity. Although MFC was two times more sensitive to PD detection than the PZT sensor, PZT presents a higher frequency response band.

\section{Conclusion}

One most critical issue in the transformer's operation is partial discharges, leading the device to total failure. In this sense, both industry and science have sought to develop new sensing methodologies to avoid complete losses caused by PD activity. This article applies the acoustic emission technique to perform PD detection, comparing the microfiber composite sensor (MFC) and the lead zirconate titanate (PZT). Although PZT has a higher frequency band, the sensitivity of the MFC is higher than PZT. These sensors have low cost, and the MFC is flexible, allowing its attachment to the transformer's uneven surfaces. Future works need to investigate the usefulness of the transducers to perform a PD type separation and PD localization.

Acknowledgments: The authors would like to thanks São Paulo State University (UNESP), School of Engineering, Bauru, Department of Electrical Engineering, Process IC-Unesp-58128

Conflicts of Interest: The author(s) declare(s) that there is no conflict of interest.

\section{References}

1. Rodríguez-Serna, J.M.; Albarracín-Sánchez, R.; Garnacho, F.; Álvarez, F.; Ortego, J. Partial Discharges Measurements for Condition Monitoring and Diagnosis of Power Transformers: A Review. In Proceedings of the 2019 6th International Advanced Research Workshop on Transformers (ARWtr), Córdoba, Spain, 6-9 Ocotber 2019; pp. 83-88.

2. Castro, B.; Clerice, G.; Ramos, C.; Andreoli, A.; Baptista, F.; Campos, F.; Ulson, J. Partial discharge monitoring in power transformers using low-cost piezoelectric sensors. Sensors 2016, 16, 1266. 
3. Stone, G.C. Partial discharge. VII. Practical techniques for measuring PD in operating equipment. IEEE Electr. Insul. Mag. 1991, 7, 9-19.

4. Albarracín-Sánchez, R.; Álvarez-Gómez, F.; Vera-Romero, C.A.; Rodríguez-Serna, J.M. Separation of Partial Discharge Sources Measured in the High-Frequency Range with HFCT Sensors Using PRPD-teff Patterns. Sensors 2020, 20, 382.

5. Romano, P.; Imburgia, A.; Ala, G. Partial discharge detection using a spherical electromagnetic sensor. Sensors 2019, 19, 1014.

6. Yaacob, M.M.; Alsaedi, M.A.; Rashed, J.R.; Dakhil, A.M.; Atyah, S.F. Review on partial discharge detection techniques related to high voltage power equipment using different sensors. Photonic Sens. 2014, 4, 325-337.

7. Zachariades, C.; Shuttleworth, R.; Giussani, R.; MacKinlay, R. Optimization of a high-frequency current transformer sensor for partial discharge detection using finite-element analysis. IEEE Sens. J. 2016, 16, 7526-7533.

8. Markalous, S.M.; Tenbohlen, S.; Feser, K. Detection and location of partial discharges in power transformers using acoustic and electromagnetic signals. IEEE Trans. Dielectr. Electr. Insul. 2008, 15, 1576-1583.

9. Lundgaard, L.E. Partial discharge. XIII. Acoustic partial discharge detection-fundamental considerations. IEEE Electr. Insul. Mag. 1992, 8, 25-31.

10. Ardila-Rey, J.A.; Barrueto, A.; Zerene, A.; Castro, B.A.d.; Ulson, J.A.C.; Mas'ud, A.A.; Valdivia, P. Behavior of an inductive loop sensor in the measurement of partial discharge pulses with variations in its separation from the primary conductor. Sensors 2018, 18, 2324.

11. Lundyaard, L. Partial discharge-Part XIV: Acoustic partial discharge detection-practice application. IEEE Electr. Insul. Mag. 1990, 6.

12. Aulestia Viera, M.A.; Aguiar, P.R.; Oliveira Junior, P.; Alexandre, F.A.; Lopes, W.N.; Bianchi, E.C.; da Silva, R.B.; D'addona, D.; Andreoli, A. A time-frequency acoustic emission-based technique to assess workpiece surface quality in ceramic grinding with PZT transducer. Sensors 2019, 19, 3913.

13. Standard, I.; others. High-Voltage Test Techniques: Partial Discharge Measurements; IEC-60270; 2000.

14. Ma, G.; Zhou, H.; Zhang, M.; Li, C.; Yin, Y.; Wu, Y. A High Sensitivity Optical Fiber Sensor for GIS Partial Discharge Detection. IEEE Sens. J. 2019, 19, 9235-9243.

15. Iorkyase, E.T.; Tachtatzis, C.; Glover, I.A.; Lazaridis, P.; Upton, D.; Saeed, B.; Atkinson, R.C. Improving RF-based partial discharge localization via machine learning ensemble method. IEEE Trans. Power Deliv. 2019, 34, 1478-1489.

16. Ardila-Rey, J.A.; Montaña, J.; De Castro, B.A.; Schurch, R.; Covolan Ulson, J.A.; Muhammad-Sukki, F.; Bani, N.A. A comparison of inductive sensors in the characterization of partial discharges and electrical noise using the chromatic technique. Sensors 2018, 18, 1021.

17. Siegel, M.; Beltle, M.; Tenbohlen, S.; Coenen, S. Application of UHF sensors for PD measurement at power transformers. IEEE Trans. Dielectr. Electr. Insul. 2017, 24, 331-339.

18. Li, L.; Cai, H.; Han, H.; Jiang, Q.; Ji, H. Adaptive short-time Fourier transform and synchrosqueezing transform for non-stationary signal separation. Signal Process. 2020, 166, 107231.

19. De Castro, B.A.; de Melo Brunini, D.; Baptista, F.G.; Andreoli, A.L.; Ulson, J.A.C. Assessment of macro fiber composite sensors for measurement of acoustic partial discharge signals in power transformers. IEEE Sens. J. 2017, 17, 6090-6099.

Publisher's Note: MDPI stays neutral with regard to jurisdictional claims in published maps and institutional affiliations.

(C) 2020 by the authors. Licensee MDPI, Basel, Switzerland. This article is an open access article distributed under the terms and conditions of the Creative Commons Attribution (CC BY) license (http:/ / creativecommons.org/licenses/by/4.0/). 